American Chemical Society, Ind. Eng. Chem. Res., ie061244g, Supporting Information Page S1

\title{
Polymer-templated mesoporous organosilicas with two types of multifunctional organic groups
}

\author{
Oksana Olkhovyk and Mietek Jaroniec* \\ Department of Chemistry, Kent State University, Kent, Ohio 44242
}


American Chemical Society, Ind. Eng. Chem. Res., ie061244g, Supporting Information Page S2

\section{Mercury adsorption study}

Mercury adsorption was measured from aqueous solutions. A series of experiments for the $\mathrm{Hg}^{2+}$ : ligand ratios, $3: 1$ and $6: 1$, was performed using solutions of the particular $\mathrm{Hg}^{2+}$ concentrations, which were prepared by dilution of a specified volume of the mercury (II) nitrate volumetric standard, $0.145 \mathrm{~N}$ aqueous solution, to the total volume of $10 \mathrm{ml}$. In a typical experiment $0.05 \mathrm{~g}$ of the sample was equilibrated for $40 \mathrm{~min}$ with $10 \mathrm{ml}$ of mercury aqueous solution of known concentration. The filtrate was collected after adsorption and the sample was washed with $5 \mathrm{ml}$ of DI water. The collected solution was diluted up to $25 \mathrm{ml}$. All samples were dried under vacuum at room temperature for 24 hours and subsequently subjected to the thermal analysis under controlled conditions.

\section{Determination of the adsorbent capacity}

Mercury (II) concentration in the filtrate after adsorption was measured spectrophotometrically with dithizone (diphenylthiocarbazone) as a complexing agent. Mercury photometric determinations were performed on a Shimadzu-1601 spectrometer in $1 \mathrm{~cm}$ quartz photocell (volume ca. $5 \mathrm{ml}$ ). The amount of mercury was determined from the calibration curve that was prepared for the mercury concentration interval of 0-50 $\mu$ g with desirable correlation to the complexing agent concentration. The background correction was performed against pure chloroform. Each sample was analyzed at $490 \mathrm{~nm}$ wavelength.

The protocol for the calibration curve preparation and analysis of the sample was done by adjusting $\mathrm{pH}$, which promoted the stabilization of mercury dithizonate. In each measurement, a specific aliquot of mercury solution was mixed with $50 \mathrm{ml}$ of $0.1 \mathrm{~N}$ hydrochloric acid in separation funnel with subsequent addition of $5 \mathrm{ml}$ of pure chloroform and $5 \mathrm{ml}$ of $20 \%$ (w/v) of hydroxylamine hydrochloride. Mixture was shaken in separation funnel for 1 minute, and chloroform fraction discarded. $25 \mathrm{ml}$ of dithizone solution $(10 \mathrm{mg} / \mathrm{L}$, prepared daily with chloroform) and 3ml of 30\% acetic acid were added, shaken vigorously, and chloroform layer was filtered through cotton, and analyzed in quartz cuvette. 

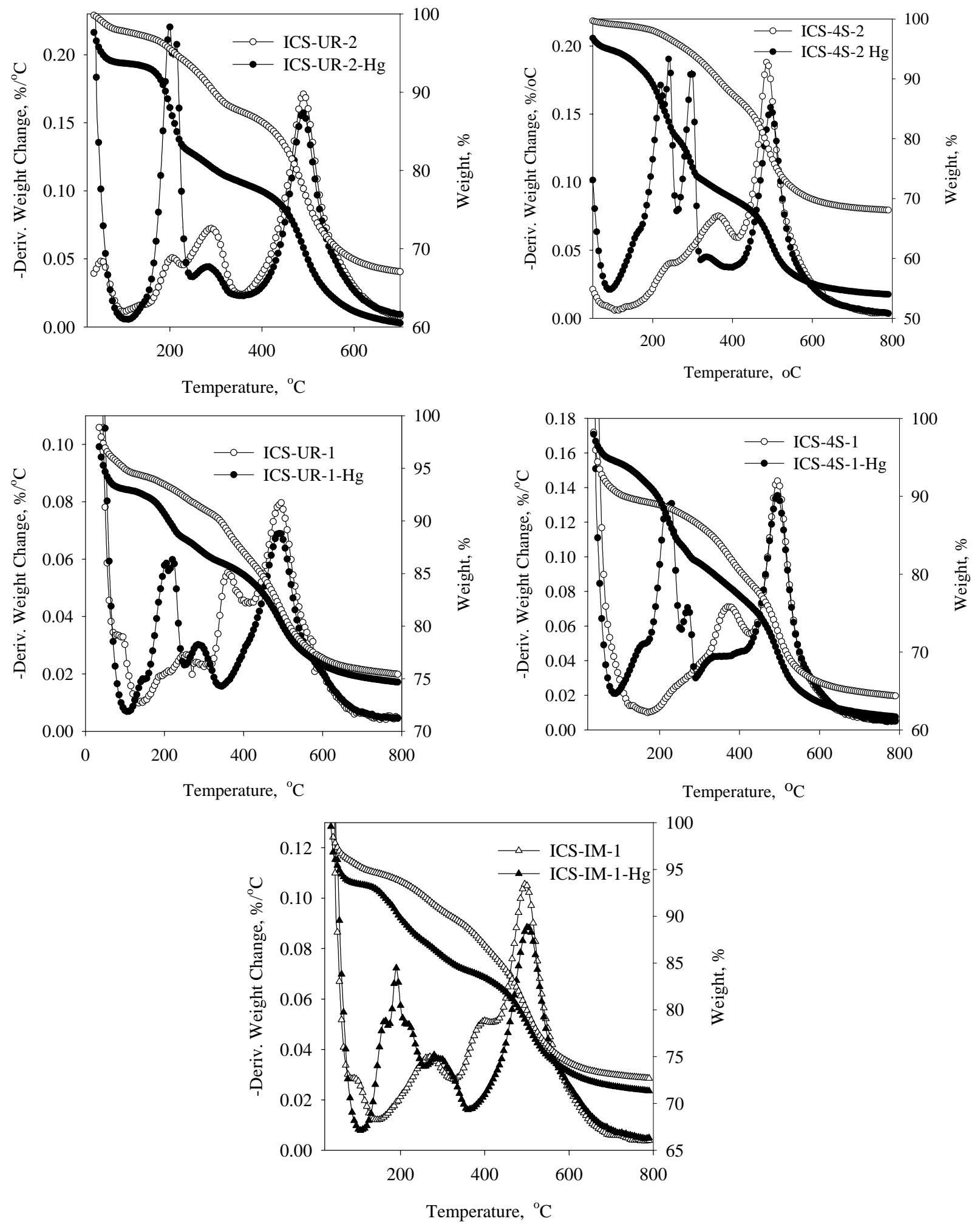

Figure 1S. Thermogravimetric (TG) and differential thermogravimetric (DTG) weight change curves for the samples studied with lower (1) or higher (2) concentration of incorporated organics before (e.g., ICS-UR-2) after mercury (e.g., ICS-4S-2-Hg) adsorption. 
American Chemical Society, Ind. Eng. Chem. Res., ie061244g, Supporting Information Page S4


Figure 2S. Differential (DTG) weight change curves for the samples studied with lower (1) and higher (2) concentration of incorporated organics after mercury adsorption tests before (e.g., ICS-UR-2) after mercury (e.g., ICS-4S-2-Hg) adsorption. 